ISSN 1794-9831

E-ISSN 2322-7028

Vol. 13 No. 1

Ene - Jun 2016

Cúcuta, Colombia

Recibido:

28 de Enero de 2016

Aceptado por

pares: 2 de Abril de 2016

Aprobado:

20 de Mayo de 2016

* Enfermera. Doctora en Enfermería. Profesora Asociada, Facultad de Enfermería, Universidad Nacional de Colombia.

Bogotá, Colombia. Correo electrónico: olchaparrod@unal. edu.co

** Enfermera. Magister en Enfermería.

Profesora Titular (p), Facultad de Enfermería, Universidad Nacional de Colombia. Bogotá, Colombia. Correo electrónico: lbarrerao@ unal.edu.co

*** Enfermera

Magister en

Enfermería. Profesora Asociada, Facultad de Enfermería, Universidad Nacional de Colombia. Bogotá, Colombia. Correo electrónico: evargasr@ unal.edu.co

**** Enfermera. Doctor en Enfermería. Profesora Auxiliar, Facultad de Enfermería, Universidad Nacional de Colombia. Bogotá, Colombia. Correo electrónico: spacarrenom@unal. edu.co

***** Descriptores en Ciencias de la Salud (DeCS), en la página http://decs.bvs.br/E/ DeCS2015_Alfab-S. htm de la Biblioteca virtual en salud del proyecto BIREME, de la Organización Mundial de la Salud y de la Organización Panamericana de la Salud.

\title{
Mujeres cuidadoras familiares de personas con enfermedad crónica en Colombia ${ }^{\dagger}$
}

\author{
Lorena Chaparro-Díaz* \\ Lucy Barrera-Ortiz** \\ Elizabeth Vargas-Rosero*** \\ Sonia Patricia Carreño-Moreno ${ }^{* * * *}$
}

\section{RESUMEN}

Objetivo: describir las características sociodemográficas y la sobrecarga en cuidadoras colombianas, y explorar la asociación existente entre la sobrecarga y las variables de edad, escolaridad, estrato, tiempo como cuidadora y número de horas que cuida al día. Materiales y Métodos: estudio descriptivo y de asociación con 1183 cuidadoras de las cinco regiones de Colombia. Se midieron las variables sociodemográficas y de sobrecarga con el cuidado, a partir de la aplicación de la Encuesta para la Caracterización del Cuidador Familiar de una Persona con Enfermedad Crónica y la Escala de Sobrecarga del Cuidador de Zarit. Resultados: el rango de edad de las cuidadoras que predomina está entre los 36 y 59 años, el nivel educativo es heterogéneo, la mitad de las cuidadoras se dedica al hogar como ocupación principal, religión predominante es la católica. El $80 \%$ cuida a la persona con enfermedad crónica desde el momento de su diagnóstico. Más de la mitad del grupo de participantes presentó ausencia de sobrecarga con el cuidado. La edad, el tiempo como cuidadora y el estrato socioeconómico tienen una asociación estadísticamente significativa pero de magnitud débil con la sobrecarga de cuidado. No se encontró una asociación significativa entre la escolaridad de las cuidadoras. Conclusiones: se encontraron asociaciones de magnitud leve entre la edad, el estrato socioeconómico, el tiempo como cuidadora y la sobrecarga del cuidado; es vital consolidar un modelo que explique las variables que inciden en la sobrecarga de cuidadoras de personas con enfermedad crónica en el país.

PALABRAS CLAVE: costo de enfermedad, cuidadores, enfermedad crónica, mujeres ${ }^{* * * * *}$.

Para citar este artículo / To reference this article / Para citar este artigo

Chaparro-Díaz L, Barrera-Ortiz L, Vargas-Rosero E, Carreño-Moreno SP. Mujeres cuidadoras familiares de personas con enfermedad crónica en Colombia. Rev. cienc. cuidad. 2016; 13(1): 72-86.

† Financiación: Este estudio fue desarrollado dentro del marco del programa para la disminución de la carga de las enfermedades crónicas no transmisibles en Colombia. Colciencias Convocatoria 537 de 2011, Contrato de financiación RC No. 366-2011, Código 110153730890 


\section{Women, family caregivers of persons with chronic diseases in Colombia}

Objective: to describe the sociodemographic characteristics and overload on Colombian female caregivers, and to explore the association between overload, with the variables of age, education, stratus, time as a caregiver and number of hours care per day. Materials and Methods: adescriptive study and association with 1183 caregivers from five regions of Colombia. Sociodemographic and care overload variables were measured through the administration of the Encuesta para la Caracterización del Cuidador Familiar de una Persona con Enfermedad Crónica and Escala de Sobrecarga del Cuidador de Zarit. Results: the age range of predominant caregivers is between 36 to 59 years, the educational level is heterogeneous, half of the caregivers is dedicated to the home as their main occupation, the predominant religion is Catholic. $80 \%$ care for the person with chronic illness from the time of diagnosis. More than half the group of participants presented absence of overload with care. Age, time as a caregiver and socioeconomic status, have a statistically significant but weak magnitude care overload association. No significant association between schooling of caregivers found. Conclusions: mild magnitude associations between age, socioeconomic stratum, time as a caregiver and care overload found, It is vital to consolidate a model that explains the variables that affect the overload of caregivers of persons with chronic diseases in the country.

KEY WORDS: cost of illness, caregivers, chronic disease, women. 
ISSN 1794-9831

E-ISSN 2322-7028 Vol. 13 No. 1

Ene - Jun 2016

Cúcuta, Colombia

\section{Cuidadores familiares mulheres de pessoas com doenças crônicas na Colômbia}

\section{RESUMO}

Objetivo: descrever as características sociodemográficas e sobrecarregar cuidadores colombianos, e explorar a associação entre a sobrecarga, com as variáveis idade, escolaridade, estrato, o tempo como um cuidador e do número de horas de cuidados por dia. Materiais e Métodos: estudo descritivo e de associação com os cuidadores 1183 uma das cinco regiões da Colômbia. variáveis sociodemográficas foram medidos e sobrecarga com cuidado a partir da aplicação do Encuesta para la Caracterización del Cuidador Familiar de una Persona con Enfermedad Crónica e do Escala de Sobrecarga del Cuidador de Zarit. Resultados: a faixa etária dos cuidadores predomina é entre 36 a 59 anos, o nível educacional é heterogêneo, metade dos cuidadores é dedicado à casa como sua principal ocupação, religião predominante é a católica. $80 \%$ de cuidados para a pessoa com doença crónica a partir do momento do diagnóstico. Mais da metade do grupo de participantes apresentaram ausência de sobrecarga com cuidado. Idade, tempo como um cuidador e nível socioeconômico, têm uma associação estatisticamente significativa, porém fraca sobrecarga de cuidados de magnitude. Nenhuma associação significativa entre a escolaridade dos cuidadores encontrados. Conclusões: associações leves magnitude entre idade, nível socioeconômico, o tempo como uma sobrecarga do cuidador e cuidados encontrado; É vital para consolidar um modelo que explica as variáveis que afetam cuidadores sobrecarregar as pessoas com doença crônica no país.

PALAVRAS-CHAVE: efeitos psicossociais da doença, cuidadores, doença crônica, as mulheres. 


\section{INTRODUCCIÓN}

$\mathrm{E}$ xiste evidencia de que el costo del cuidado de personas con enfermedad crónica en diferentes partes del mundo es alto (1-2). Por ende, en muchos casos este cuidado no se realiza por parte de personal profesional sino por familiares que asumen esta labor. Globalmente, las personas que toman la tarea de cuidar a una persona con enfermedad crónica son, en mayor medida, mujeres (3-4).

Según estudios, en Latinoamérica y específicamente en Colombia (5-7), la dinámica no es contraria a la internacional, es decir, el cuidado es asumido en mayor porcentaje por las mujeres; los sistemas de salud y los factores económicos han llevado a las mujeres a asumir el cuidado informal y no profesional de las personas con enfermedad crónica. Este fenómeno presenta tanto aspectos positivos como consecuencias negativas en las cuidadoras familiares, incluyendo la pérdida de ingresos económicos, disminución de la calidad de vida, aumento del estrés y problemas de salud (8)

En el contexto del cuidado de una persona con enfermedad crónica existen diversas características sociodemográficas como el nivel de educación, edad, sexo, religión, número de horas dedicadas al cuidado, tiempo como cuidador, habilidad de cuidado y soporte social, entre otras, que pueden influir en la experiencia de ese cuidado (9-10).

En este sentido, Espín (11) sostiene que mujeres trabajadoras, con formación académica tienen un desempeño favorable y enfrentan mejor el cuidado de su familiar. De otro lado, otros autores $(6,12)$ también han relacionado el tiempo de cuidado con la aceptación de la enfermedad, relacionando una mayor adaptación con mayor tiempo trascurrido en el cuidado y, en consecuencia, una menor carga del cuidado.

El perfil de los cuidadores familiares, específicamente en mujeres españolas, muestra que el nivel de estudios y nivel socioeconómico es menor en mujeres, generalmente ellas cuidan personas con mayor dependencia y esto también se relaciona con mayor percepción de carga asumida y peor salud física; tienen poco acceso a trabajos remunerados al mismo tiempo de la labor de cuidado y existe riesgo de sedentarismo, falta de sueño y mayor número de consultas a servicios de salud mental (13).

Otro estudio realizado en España por López y Crespo (14) especifica que no existen diferencias entre cuidadoras mujeres y hombres, específicamente en variables como la edad. Se afirma que las mujeres cuidadoras, por lo general, cuidan de sus madres, tienen más conflictos con sus roles familiares y experimentan mayor sobrecarga, consumen más medicamentos y presentan depresión. Sin embargo, entre hombres y mujeres no hay diferencia en el nivel de ansiedad, expresión de la ira y nivel de salud física.

En Colombia, las características sociodemográficas tienen una semejanza a otros contextos. La experiencia de ser cuidadora, para la mujer colombiana, representa una responsabilidad importante para casos en que el familiar enfermo es un niño porque se relaciona con ser padre o madre, cuidar durante más de 37 meses, dedicarse a labores del hogar y estar en unión libre. En cuidadores de adultos se asocia a cuidar pacientes mayores, llevar 36 meses o menos, ser hijo del paciente y estar casado (15).

El estrato socioeconómico no representa sobrecarga en cuidadores de Alzheimer (15) y el género no indica mayores diferencias de la experiencia (16). El impacto de la enfermedad afecta la salud del cuidador, específicamente en antecedentes de enfermedades, sensación de salud, fatiga y dolores musculares (17).

La calidad de vida se ve afectada específicamente en las dimensiones física, sicológica y social (18-20); aunque en cuidadores de niños, el más afectado es el bienestar psicológico (21). No existen niveles de significancia importantes entre la calidad de vida y el nivel de funcionalidad del paciente, pero sí entre la dimensión de bienestar psicológico y los aspectos de funcionalidad de miembros superiores, función excretora y socialización (22); entre la calidad de vida y el género, la ocupación y la relación con el paciente (19) y con el nivel socioeconómico y ser único cuidador (23).

El bienestar social se relaciona con ser único cuidador (23). Cuidar de un adulto y un niño afecta de forma particular la calidad de vida. Los aspectos más afectados de cuidar de un adulto son el bienestar psicológico y social, mientras que cuidar de un niño

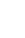


ISSN 1794-9831

E-ISSN 2322-7028

Vol. 13 No. 1

Ene - Jun 2016

Cúcuta, Colombia afecta más el bienestar espiritual. El bienestar físico se ve afectado de forma similar en ser cuidador de adulto o niño (24).

Cuidar una persona con Enfermedad Crónica no Transmisible (ECNT) y otra con enfermedad crónica transmisible muestra aspectos significativos en situaciones de ECNT con más presencia de cuidadores que cuidan desde el diagnóstico, cuidar una persona del mismo rango del cuidador, tener más de 35 años en el cuidador, dedicarse al hogar, el nivel socioeconómico bajo, llevar como cuidador más de 18 meses y dedicarse más de 12 horas y cuidar a un familiar consanguíneo (hijo) (25).

La sobrecarga es un aspecto medular en la predicción de los aspectos negativos de la experiencia. El estudio de Manso et al. (26) indica que la poca funcionalidad del paciente, las horas dedicadas al cuidado, la ansiedad, la depresión y la salud psíquica son moduladores de la sobrecarga moderada en cuidadoras mujeres, lo que reafirma la condición de vulnerabilidad, especialmente en zonas rurales, en donde existe un menor acceso al desarrollo científico, menor transporte público y horarios de trabajo irregulares.

Aldana (27), en una muestra de 220 cuidadores, encontró que la mayor sobrecarga está relacionada con la baja percepción de salud y el incremento en la somatización y ansiedad.

Estudios en Colombia indican que la sobrecarga se relaciona y asocia con el género (femenino), edad (mayor edad del proveedor de cuidado), ocupación hogar, estado civil (casados), horas dedicadas al cuidado (mayor tiempo) y la certeza sobre el futuro $(28,29)$.

En Colombia la sobrecarga en cuidadores ha sido valorada en algunas regiones del país y muestra una baja sobrecarga en regiones como Orinoquia, Caribe insular y continental y Amazónica $(30,31)$, mientras que en el Pacifico hay una sobrecarga intensa del $75 \%$ (32).

Estas características han sido descritas parcialmente en Colombia (33), donde adicionalmente se ha observado la habilidad de cuidado, el soporte social y el uso de tecnologías de la información y la comunicación que estos cuidadores tienen (34). Pese a existir avances en la caracterización sociodemográfica de cuidadoras familiares y evaluación de la sobrecarga del cuidado, éstos proceden de pequeños grupos homogeneos en zonas urbanas que no incluyen el amplio rango de escenarios que tiene el país.

$\mathrm{Al}$ tener una descripción de las mujeres cuidadoras en diferentes condiciones socioeconómicas, en todo el territorio colombiano, se pretende no sólo acercarse a la realidad de esta población para el desarrollo de programas y políticas, sino establecer la asociación de algunas características sociodemográficas con la sobrecarga generada en el cuidador de la persona con enfermedad crónica.

\section{MATERIALES Y MÉTODOS}

Estudio descriptivo y de asociación, con cuidadoras familiares de personas con enfermedad crónica de las 5 regiones del país, con datos recolectados durante el año 2013 en el marco de una muestra maestra en el Programa para la disminución de la carga de la enfermedad crónica no transmisible (ECNT) en Colombia. La muestra fue intencional, y estuvo constituida por 1.183 cuidadoras, con distribución por regiones: Región Amazonía (4,1 \%), Andina (79,6\%), Caribe (7,5\%), Orinoquía (3,5\%), Pacifica $(5,3 \%)$. Los $n$ se depuraron a partir de la base de datos del programa para la disminución de la carga de la ECNT. 
Tabla 1. Muestra del estudio (intencional).

\begin{tabular}{|l|r|r|}
\hline Región & $\mathbf{n}$ & $\mathbf{\%}$ \\
\hline Amazonía & 49 & 4,1 \\
\hline Andina & 942 & 79,6 \\
\hline Caribe & 89 & 7,5 \\
\hline Orinoquía & 41 & 3,5 \\
\hline Pacífica & 63 & 5,3 \\
\hline Total & 1183 & 100 \\
\hline
\end{tabular}

Fuente: Base de datos del estudio.

El analisis por regiones de la población, realizado a partir de datos del Ministerio de Salud y el Departamento Nacional de Estadisticas (DANE) (35) para el 2012, muestra que en la región Andina se encuentra el $61,86 \%$ de la población colombiana y es el máximo porcentaje a nivel nacional, lo que corresponde con la muestra de este estudio. Adicionalmente, la mayoria son mujeres (50.7\%), La mortalidad asociada a ECNT por regiones tambien da muestra del mayor porcentaje en la región Andina, con un 47,6 \%. Esto sustenta el hecho de que las diferencias de muestra presenten mayor presencia de cuidadores familiares en la región Andina.

Las participantes fueron captadas en las instituciones prestadoras de servicios de salud de las distintas regiones, en el marco del programa para la disminución de la Carga de la Enfermedad crónica en Colombia, a partir de muestra maestra, lo que implicó la obtención de los avales institucionales correspondientes. Se incluyeron cuidadoras que tuvieran más de un mes en su actividad de cuidado al paciente crónico, mayores de 18 años o menores con el consentimiento de un adulto, sin dificultades para responder la entrevista de caracterización y percepción de carga con el cuidado y que aceptaran participar en el estudio.

La recolección de los datos se realizó a través de nodos de la Red Latinoamericana de Cuidado al paciente crónico y la Unión Temporal Carga de la Enfermedad crónica No Transmisible en Colombia; cada nodo contaba con un equipo de investigación que recibió la capacitación en el diligenciamiento de los instrumentos y acompañamiento permanente a través de videoconferencias, comunicaciones electrónicas y sesiones presenciales.

Se midieron las variables sociodemográficas tales como edad, escolaridad, estado civil, ocupación, religión, estrato socioeconómico, cuidado desde el momento del diagnóstico de la enfermedad crónica, tiempo como cuidador, apoyos que tiene el cuidador, número de horas diarias dedicadas al cuidado $\mathrm{y}$ relación con la persona cuidada, por medio de la Encuesta para la Caracterización del Cuidador Familiar de una Persona con Enfermedad Crónica que se deriva a la Encuesta de Caracterización de la Diada elaborada por el autor principal de este reporte. Esta encuesta contó con validez aparente, comprensibilidad y validez de expertos (36); a través de la escala de Sobrecarga del Cuidador de Zarit (37) se midió la Sobrecarga del Cuidador.

El instrumento de sobrecarga mide multidimensionalmente el fenómeno de sobrecarga del cuidador y consta de 22 ítems con escala tipo Likert de 5 opciones (Nunca, Rara vez, algunas veces, bastantes veces y casi siempre). La puntuación va desde 22 a 110 puntos y la sobrecarga se puntúa: Ausencia de Sobrecarga ( $<$ o igual a 46), Sobrecarga Ligera (47 a 55), Sobrecarga Intensa ( $>$ o igual a 56). Este instrumento cuenta con pruebas psicométricas en español, una validez de 0.92 y una confiabilidad de 0.81 (38). Los dos instrumentos fueron autorizados por sus autores para ser utilizados.

Se usó estadística descriptiva para el análisis de las variables sociodemográficas; la asociación entre las variables se realizó a través de la prueba de asociación d de Somers para variables con nivel ordinal. La prueba Chi Cuadrado no fue utilizada debido a que las frecuencias esperadas fueron menores a 5 . La información recogida fue analizada mediante el programa SPSS $^{\circledR}$ (SPSS, versión 22.0 para Windows).

Este estudio fue aprobado por el Comité de Ética institucional como parte del Programa para disminuir la carga de la enfermedad crónica en 
ISSN 1794-9831

E-ISSN 2322-7028

Vol. 13 No. 1

Ene - Jun 2016

Cúcuta, Colombia
Colombia y mantiene todos los requisitos exigidos por la Resolución No. 8430 de 1993 (39), por la cual se establecen las normas científicas, técnicas y administrativas para la investigación en salud en Colombia, y la aplicación de los principios éticos para la investigación y lo definido en la Ley 911 de 2004 (40) que orienta el ejercicio ético de enfermería. Cada participante firmó el consentimiento informado de la investigación.

\section{OBJETIVOS}

\section{Objetivo general}

Describir las características socio demográficas y la sobrecarga en cuidadoras colombianas.

\section{Objetivos específicos}

- Definir el perfil sociodemográfico y de cuidado de las cuidadoras familiares mujeres.

- Determinar la asociación existente entre la sobrecarga y las variables de edad, escolaridad, estrato, tiempo como cuidadora y número de horas que cuida al día.

\section{RESULTADOS}

Entre las limitaciones del estudio se encuentra que los cuidadores, al no ser reconocidos socialmente, debieron ser abordados únicamente a nivel institucional hospitalario a través del paciente, lo que requirió mayor tiempo en el proceso de recolección de información; también los múltiples entrevistadores a nivel del país puede ser un condicionante de los resultados. En cuanto al diligenciamiento de los instrumentos, la valoración de la sobrecarga representó mayor esfuerzo en las cuidadoras.

\section{Caracterización sociodemográfica}

Respecto a las variables sociodemográficas, el rango de edad que predomina en las cuidadoras se ubica entre 36 y 59 años en el 49,4\%, seguido por el 28,7\% con edades comprendidas entre los 18 y los 35 años. En cuanto al estado civil, son cuidadoras casadas el $33,3 \%$, solteras el 31,2 \% y en unión libre el 21,2 $\%$. Claramente se aprecia que las mujeres en edad productiva deben ejercer el cuidado y que se asume que es parte innata de su rol; sin embargo, se aprecia mayor vulnerabilidad y riesgo de insuficiencia del rol por la sobrecarga de funciones aparte de la labor de cuidado, como la formación educativa y las relaciones de pareja existentes. En la Tabla 1 se describen las características sociodemográficas de las cuidadoras. 
Tabla 1. Caracterización cuidadoras familiares en las 5 regiones de Colombia.

\begin{tabular}{|c|c|c|c|c|c|}
\hline Variable & Frecuencia & Porcentaje & Variable & Frecuencia & Porcentaje \\
\hline \multicolumn{3}{|l|}{ Escolaridad } & \multicolumn{3}{|c|}{ Estrato socioeconómico } \\
\hline Analfabeta & 12 & 1 & 1 & 336 & 28,4 \\
\hline Primaria Incompleta & 170 & 14,4 & 2 & 417 & 35,2 \\
\hline Primaria Completa & 134 & 11,3 & 3 & 324 & 27,4 \\
\hline Bachillerato Incompleto & 217 & 18,3 & 4 & 85 & 7,2 \\
\hline Bachillerato Completo & 253 & 21,4 & 5 & 14 & 1,2 \\
\hline Técnico & 182 & 15,4 & 6 & 4 & 0,3 \\
\hline Universidad Incompleta & 68 & 5,7 & Indígena & 3 & 0,3 \\
\hline Universidad Completa & 120 & 10,1 & \multicolumn{3}{|c|}{ Cuida a la persona desde el diagnóstico } \\
\hline Postgrados & 27 & 2,3 & Si & 946 & 80 \\
\hline \multicolumn{3}{|l|}{ Edad } & No & 237 & 20 \\
\hline Menor de 18 años & 20 & 1,7 & \multicolumn{3}{|c|}{ Tiempo como cuidador } \\
\hline de 18 a 35 años & 340 & 28,7 & 0 a 6 meses & 243 & 20,5 \\
\hline de 36 a 59 años & 584 & 49,4 & 7 a 18 meses & 244 & 20,6 \\
\hline de 60 a 75 años & 206 & 17,4 & 19 a 36 meses & 178 & 15 \\
\hline Más de 75 años & 33 & 2,8 & Más de 36 meses & 518 & 43,8 \\
\hline \multicolumn{3}{|l|}{ Estado civil } & \multicolumn{3}{|c|}{ No. de horas que cuida al día } \\
\hline Soltero & 369 & 31,2 & Menos de 6 horas & 363 & 30,7 \\
\hline Casado & 394 & 33,3 & 7 a 12 horas & 333 & 28,1 \\
\hline Separado & 100 & 8,5 & 13 a 23 horas & 169 & 14,3 \\
\hline Viudo & 69 & 5,8 & 24 horas & 318 & 26,9 \\
\hline Unión libre & 251 & 21,2 & \multicolumn{3}{|l|}{ Único cuidador } \\
\hline \multicolumn{3}{|l|}{ Ocupación } & si & 543 & 45,9 \\
\hline Hogar & 554 & 46,8 & no & 640 & 54,1 \\
\hline Empleado & 246 & 20,8 & \multicolumn{3}{|c|}{ Apoyos con los que cuenta } \\
\hline Independiente & 204 & 17,2 & Familia & 607 & 51,3 \\
\hline Estudiante & 59 & 5 & Espiritual & 67 & 5,7 \\
\hline Otro & 120 & 10,1 & Social & 212 & 17,9 \\
\hline \multicolumn{3}{|l|}{ Religión } & Ninguno & 297 & 25,1 \\
\hline Católica & 947 & 80,1 & \multicolumn{3}{|c|}{ Relación con la persona cuidada } \\
\hline Cristiana & 159 & 13,4 & Esposa & 193 & 16,3 \\
\hline Otras & 69 & 5,8 & Madre & 307 & 26 \\
\hline Ninguna & 8 & 0,7 & Hija & 361 & 30,5 \\
\hline & & & Abuela & 22 & 1,9 \\
\hline & & & Amiga & 43 & 3,6 \\
\hline & & & Otro & 257 & 21,7 \\
\hline
\end{tabular}

Fuente: Encuesta de Caracterización de la Diada (sección cuidador familiar), 2013. 
El 21,4 \% de las cuidadoras ha terminado su educación secundaria, seguido por el 18,3\% que tiene la educación secundaria sin finalizar y formación técnica en un $15,4 \%$; en los extremos se encuentra el $1 \%$ de las cuidadoras analfabeta y el 2,3 $\%$ que tienen título de posgrado. Adicional a estos resultados, en cuanto al acceso a la educación que han tenido las cuidadoras mujeres, se aprecian otros riesgos importantes para el proyecto de vida, puesto que, en su mayoría, tienen niveles de educación no concluidos que limitan el acceso laboral y las oportunidades, especialmente para el momento en que deseen vincularse a un medio laboral (ver tabla $1)$.

Aproximadamente la mitad de las cuidadoras (46,8 $\%$ ) se dedica al hogar como ocupación principal; luego se encuentra el 20,8 \% que son empleadas y, en menor porcentaje, estudiantes con el $5 \%$. La religión predominante es la católica en el 80,1\%. El 35,2 $\%$ de las participantes se encuentran en el estrato socioeconómico 2, seguido por cuidadoras que se ubicaron en los estratos 1 y 3 entre el 28 y $27 \%$, respectivamente.

La labor de cuidado concerniente en gran medida con los aspectos domésticos siempre se relacionará con el hogar como la ocupación de la mujer; sin embargo, se aprecia un multi-rol al considerar otras opciones como el trabajo independiente y el trabajo formal, que pueden generar alternativas para mejorar los ingresos. Además, Colombia, como país con alta influencia católica, refleja esta tendencia en las cuidadoras mujeres. El nivel socioeconómico valorado por el estrato de la vivienda es un elemento muy importante porque demuestra la situación de vulnerabilidad social que tiene la mujer y, sobre todo, cuando es cuidadora (ver Tabla 1).

Respecto a la experiencia del cuidado, se encontró que el $80 \%$ de las participantes cuidan a la persona con enfermedad crónica desde el momento de su diagnóstico y es la única cuidadora en el 45,9\% de los casos. En el tiempo de cuidado, el 43,8 \% lleva más de 36 meses, seguido por 20,6\% que lleva entre 7 y 14 meses. Aproximadamente una tercera parte de las participantes $(30,7 \%)$ cuida a la persona menos de seis horas. El tiempo de cuidado en términos de prolongación y cantidad en horas, meses y años, es un aspecto poco reconocido en la labor de las cuidadoras mujeres; en este estudio se tiene un parámetro importante de la dedicación que permite predecir aspectos sobre la sobrecarga (ver Tabla 1).

Existe una relación de parentesco en primer grado de consanguinidad con la persona cuidada: de hija en el $30,5 \%$, de madre en el $26 \%$ y de esposa en el $16,3 \%$ de los casos, y apoyo familiar para las cuidadoras en más de la mitad de participantes (51,3\%). La familia y el rol que tiene la mujer dentro de ésta con la atención de los procesos del ciclo vital y de salud-enfermedad ha hecho que el valor del cuidado sea un aspecto vital para el mantenimiento de lazos familiares y un apoyo social efectivo.

\section{Sobrecarga de cuidado}

En relación con la sobrecarga de la cuidadora, más de la mitad del grupo de participantes (67\%) presentó ausencia de sobrecarga, una sobrecarga ligera el $25 \%$ y sobrecarga intensa el $8 \%$.

\section{Asociación entre variables}

Para determinar la existencia de asociación entre las variables sociodemográficas edad, escolaridad, estrato socioeconómico, tiempo como cuidadora y número de horas que cuida al día, y la variable sobrecarga de cuidado, se realizó la prueba estadística $\mathrm{d}$ de Somers. Al respecto, la edad (-0.09) y el estrato socioeconómico (-0.066) tienen una asociación estadísticamente significativa, pero de magnitud débil con la sobrecarga de cuidado. El tiempo como cuidadora, en meses, tiene una asociación débil y estadísticamente significativa con el nivel de sobrecarga. Por otra parte, no se encontró una asociación significativa entre la escolaridad de las cuidadoras y el número de horas que cuidan al día con la sobrecarga (ver Tabla 2). 
Tabla 2. Asociación de variables sociodemográficas con la sobrecarga del cuidado d de Somers.

\begin{tabular}{|l|l|l|l|l|l|l|l|l|l|l|}
\hline \multicolumn{2}{|l|}{ Edad } & \multicolumn{3}{|l|}{ Escolaridad } & \multicolumn{2}{l|}{ Estrato Socioeconómico } & \multicolumn{2}{l|}{ Tiempo como cuidadora } & \multicolumn{2}{l|}{ Número de horas que cuida al día } \\
\hline D & Valor p & D & Valor p & D & Valor p & D & Valor p & D & Valor p \\
\hline-0.09 & $<0.001$ & 0.014 & 0.466 & $-0,066$ & 0.002 & 0.109 & $<0.001$ & 0.028 & 0.189 \\
\hline
\end{tabular}

Fuente: Encuesta de caracterización de la diada (sección cuidador familiar) - Encuesta de sobrecarga del cuidado de Zarit, 2013.

\section{DISCUSIÓN}

Los mayores porcentajes de escolaridad de las participantes se ubicaron en el bachillerato incompleto y completo (18\% y $21 \%)$, datos que se corresponden con cifras publicadas por el Departamento Nacional de Estadísticas (DANE) (41) en el año 2012. De igual manera, se encuentra un porcentaje de analfabetismo en mujeres del $1 \%$, que es mucho más bajo que el promedio nacional en mujeres, el cual se ubica en $6,56 \%(42)$.

El analfabetismo en cuidadores familiares en las diferentes regiones del país no ha superado el $2 \%$ en las regiones Amazónica, Pacifica y Caribe (30-32, 43). Este hallazgo se puede explicar si se observa que el mayor porcentaje de las cuidadoras se ubica en la franja de los 18 a 59 años, población que ha tenido más facilidad de acceso a la educación en Colombia (42).

Es preocupante, con respecto a la edad de las participantes, que el $78,1 \%$ de las participantes pertenezca al grupo de población económicamente activa y que casi la mitad (46,8 \%), tenga como principal ocupación el hogar. Si se analizan estos datos desde la realidad del contexto colombiano, se encuentra que el porcentaje de desempleo en las mujeres, para el 2013 , fue de $11 \%$, cifra muy superior al $6,4 \%$ de los hombres.

En Colombia, la edad de los cuidadores familiares de las regiones Amazonía, Orinoquia, Pacifica y Caribe oscila entre 36 y 59 años, seguida de cuidadores entre 18 y 35 años, lo que confirma menores posibilidades de acceso laboral estable por tener que atender la labor de cuidado. $(30-32,43)$.

Según un estudio reciente del DANE (41), el estar a cargo de una persona que necesite cuidado en el hogar, disminuye la tasa de ocupación en 10,3 puntos porcentuales respecto a aquellas mujeres que no lo tienen. Lo anterior, aunado al papel que ha desempeñado históricamente la mujer en el cuidado de los enfermos (44) y la falta de inclusión laboral, podría explicar la cifra de $46,8 \%$ de cuidadoras, cuya principal ocupación es el hogar.

En relación con la ocupación de los cuidadores familiares al nivel de las regiones Amazonía, Pacifica, Caribe y Orinoquia se aprecia que la ocupación en el hogar es una variable predominante; sin embargo, las regiones Amazonia, Caribe Insular y Orinoquia presentan un incremento en al menos 10 puntos porcentuales con respecto a este estudio, en actividades remuneradas tipo empleado y trabajador independiente (entre $45 \%$ y $56 \%)(30-32,43)$.

Las mujeres cuidadoras de este estudio son, en su mayoría, las hijas de las personas con enfermedad crónica, dato que es similar al encontrado por el estudio de la Alianza Nacional para el Cuidado en Estados Unidos (4), donde el $36 \%$ de los cuidadores cuidaba a sus padres, así como con lo reportado en la región Orinoquía (31) y Caribe (30). El hallazgo relacionado con que la tarea del cuidado de la persona con enfermedad crónica recaiga en un hijo y que exista un apoyo familiar en la mitad de los casos, puede estar dado por la responsabilidad y el amor filial que existe entre padres e hijos, tal como lo explican varios estudios (45-47), y por la unión familiar característica en la cultura colombiana (48). Sin embargo, para la región de la Amazonía (43) se presenta un panorama similar a lo encontrado en este estudio con respecto a tener una relación de madre, lo cual es muy común en el perfil de cuidadoras mujeres.

De acuerdo con el estado civil de las cuidadoras, el 
ISSN 1794-9831

E-ISSN 2322-7028

Vol. 13 No. 1

Ene - Jun 2016

Cúcuta, Colombia
$54,5 \%$ de ellas son casadas o viven en unión libre. Varios estudios $(49-50,31)$ han confirmado el apoyo que representa para un cuidador tener un vínculo o lazo afectivo, ya que tener una pareja favorece el desahogo, la catarsis y el apoyo en la toma de decisiones en el cuidado. Sin embargo, esta afirmación no es válida en aquellos cuidadores que cuidan a su pareja, puesto que en estos existe una dinámica diferente (51). Por otro lado, la tendencia también es a que el cuidador pueda disponer de toda su energía personal siendo soltero para ejercer el cuidado (43).

Otras características que se han encontrado en varios estudios $(30-32,43)$ en las regiones Pacífica, Amazónica, Orinoquía y Caribe corresponden con la dedicación que, en su mayoría, es al hogar, rol asumido históricamente por la mujer y que genera socialmente una invisibilidad del cuidado en la cronicidad.

En cuanto al tiempo de cuidado, los resultados de este estudio difieren en gran medida con los encontrados en la región Amazónica (43), en donde la dedicación es superior a 37 meses en mayor porcentaje ( $76 \%)$. En el cuidado es común no encontrar límites en cuanto a tiempo y dedicación, lo que ocasiona un sub-registro.

La dedicación en horas de las cuidadoras familiares de este estudio reporta que al menos el $50 \%$ del día (que equivale $100 \%$ al tiempo vespertino de una persona) es dedicado al cuidado $(30-32,43)$, lo que muestra sobrecarga en tiempo y pérdida de productividad social de un país, al reemplazar el desarrollo personal a través del estudio y el trabajo por la labor de cuidado. Estos resultados indican la necesidad de reconocimiento social y económico de la labor de cuidado y más en situaciones de cronicidad.

Un dato muy llamativo de este estudio y del realizado en el Amazonas (43) y Caribe (30) es el hecho de no ser el único cuidador con porcentajes considerables $(54,1 \%)$, lo que indica que la labor de cuidado puede ser asumida por otras mujeres del hogar o se vienen involucrando más actores.

La ausencia de sobrecarga en las cuidadoras fue un hallazgo relevante que se encontró en el $67 \%$ de las participantes, en contraste con la presencia de sobrecarga ligera e intensa, que ocupó el $25 \%$ y 8 $\%$ respectivamente. Cabe señalar que Ocampo et al.
(52), en el 2007, también encontraron en una muestra de cuidadores de la región Pacífica una ausencia de sobrecarga con un $54.2 \%$. Adicionalmente, Arias et al. (30), en un estudio reciente de cuidadores en las regiones colombianas de frontera, mostró un comportamiento similar con ausencia de sobrecarga en 4 de las 5 regiones estudiadas.

En varias investigaciones (52-56), la sobrecarga estuvo disminuida, en parte, por el apoyo social y la posibilidad de dar un respiro en las labores del cuidado, lo que podría tener similitud, en este estudio, con los apoyos familiares y en el hecho de no ser el único cuidador de la persona con enfermedad crónica. Aunque investigaciones como la de Bhattacharjee (57) no muestran una asociación estadísticamente significativa entre estas variables, sí se describe una relación entre la edad joven $(<45$ años) y el estrés del cuidador.

En virtud de lo anterior, se infiere que cuidar a una persona con enfermedad crónica, cuando se es adulto, puede interferir con momentos importantes en el desarrollo del ciclo vital como formar una familia, tener hijos o conseguir un empleo, lo que puede ocasionar frustración y sobrecarga.

La variable estrato socioeconómico tiene el mismo comportamiento que la edad; es posible que a mayores recursos económicos sea menor el nivel de sobrecarga, coincidiendo con otros estudios (58). Así mismo, es de señalar que en el contexto colombiano el alto costo de la enfermedad crónica y el escaso soporte económico del sistema de salud lleva a las cuidadoras a hacerse cargo de la compra de insumos, medicinas y terapias alternativas para el cuidado de los pacientes con enfermedad crónica.

La escolaridad, por su parte, no presentó relación alguna con la sobrecarga, resultado que es soportado por el estudio de Flores (58). La inferencia sobre este resultado es que, independientemente del nivel de educación que tengan las cuidadoras, la sobrecarga las afecta de la misma forma. Al respecto, Giraldo y Franco (8) describen la afectación que sufren los cuidadores familiares con diferentes niveles educativos en la calidad de vida y como una consecuencia de su rol.

Mientras que el número de horas al día dedicadas 
al cuidado de la persona con enfermedad crónica no tiene relación con la sobrecarga, el tiempo como cuidador en meses sí presenta una asociación con el nivel de sobrecarga. En otros estudios $(54,60)$ se ha documentado la asociación de las dos variables con la sobrecarga. Ahora bien, el tiempo como cuidador, en años, tiene relación con la carga, aspecto que es ratificado en investigaciones donde se describe que la acumulación de situaciones y emociones a través del tiempo terminan por generar estrés y carga en el cuidador $(8,60)$.

\section{CONCLUSIONES}

Las cuidadoras desarrollan su rol en edades productivas entre los 18 y 59 años con un porcentaje de $78,1 \%$; predomina el estado civil casada y el nivel educativo básico a medio. La ocupación principal es el hogar (46,8 \%), la creencia religiosa en la mayoría es la católica. Se destaca el estrato socioeconómico 2 en los participantes.

Respecto a las características del cuidado, más del $80 \%$ cuida a la persona a su cargo desde el momento de su diagnóstico, el 43,8 \% lleva más de 36 meses desempeñando la labor del cuidado y no siempre son las únicas cuidadoras. La mayoría de las cuidadoras presenta una relación en primer grado de consanguinidad con la persona a la que cuidan. Los apoyos que las cuidadoras mujeres reportan son, en primera medida, el familiar, y en segunda instancia, el social.

Con respecto a la sobrecarga, la mayoría de las cuidadoras $(67 \%)$ presenta ausencia de sobrecarga, seguido por sobrecarga ligera en un $65 \%$.

Se encontraron asociaciones de magnitud leve entre la edad, el estrato socioeconómico, el tiempo como cuidadora y la sobrecarga del cuidado. Estos hallazgos aportan información contundente para la descripción del fenómeno del cuidado informal de personas con enfermedad crónica en Colombia y evidencian la necesidad de consolidar un modelo que explique las variables que inciden en la sobrecarga de cuidadoras de personas con enfermedad crónica en el país, además de reafirmar la importancia de crear programas gubernamentales destinados a dar reconocimiento y apoyo a las mujeres cuidadoras que se dedican a esta labor.

\section{CONFLICTO DE INTERESES}

Los autores declaran no tener ningún conflicto de intereses.
ISSN 1794-9831

E-ISSN 2322-7028 Vol. 13 No. 1

Ene - Jun 2016

Cúcuta, Colombia 
ISSN 1794-9831

E-ISSN 2322-7028 Vol. 13 No. 1

Ene - Jun 2016 Cúcuta, Colombia

\section{REFERENCIAS BIBLIOGRÁFICAS}

1. Feinberg L, Reinhard SC, Houser A, Houser A, Choula R. Valuing the invaluable: 2011 update - the growing contributions and costs of family caregiving. Washington D.C.: AARP Public Policy Institute; 2011.

2. Vaingankar JA, Subramaniam M, Picco L, Eng GK, Shafie S, Sambasivam R, et al. Perceived unmet needs of informal caregivers of people with dementia in Singapore. Int Psychogeriatr. [Internet]. 2013 [consultado 10 de octubre de 2015]; 25: 1605-1619. Disponible en: doi: 10.1017/S1041610213001051.

3. Trivedi R, Beaver K, Bouldin E, Eugenio E, Zeliadt SB, Nelson K, et al. Characteristics and well-being of informal caregivers: Results from a nationally-representative US survey. Chronic Illn. [Internet]. 2013[consultado 10 de octubre de 2015]; 10:167-179. Disponible en: doi:10.1177/1742395313506947

4. National Alliance for Caregiving, AARP. Caregiving in the U.S. Bethesda: National Alliance for Caregiving and AARP, 2009.

5. Flores E, Rivas E, Seguel F. Nivel de sobrecarga en el desempeño del rol del cuidador familiar de adulto mayor con dependencia severa. Ciencia y Enfermería 2012; 18(1): 29-41.

6. Pérez-Perdomo M, Llibre-Rodrguez JJ. Características sociodemográficas y nivel de sobrecarga de cuidadores de ancianos con Enfermedad de Alzheimer. Rev Cubana Enferm 2010; 26(3): 110-122.

7. Barrera L, Carrillo GM, Chaparro L, Pinto N, Rodríguez A, Sánchez B. Effect of the Program Caring for caretakers: Findings of a multicenter study. ColombMed 2011; 42: 35-44.

8. Giraldo MC, Franco AG. Calidad de vida de los cuidadores familiares. Aquichan 2006; 1: 38-53.

9. Marrón EM, Redolar-Ripoll D, Boixadós M, Nieto R, Guillamón N, Hernández E, et al. Burden on Caregivers of Children with Cerebral Palsy: Predictors and Related Factors. Univ. Psychol. [Internet] 2013; [consultado 7 de enero de 2012]; 12:1-22. Disponible en: http://revistas.javeriana.edu.co/index.php/revPsycho/article/ viewFile/1928/5800

10. Kim H, Chang MD, Rose K, Kim S. Predictors of caregiver burden in caregivers of individuals with dementia. J AdvNurs. [Internet]. 2012 [consultado 10 de octubre de 2015]; 68: 846-855. Disponible en: http://www.readcube. com/articles/10.1111\%2Fj.1365-2648.2011.05787.x?r3_referer=wol\&tracking_action=preview_click\&show_ checkout $=1 \&$ purchase_referrer $=$ onlinelibrary.wiley.com\&purchase_site_license $=$ LICENSE_DENIED

11. Espín AM. Caracterización psicosocial de cuidadores informales de adultos mayores con demencia. Rev Cubana de Salud Pública 2008; 34(3): 0-0.

12. Bengozar Torres MC, Serra Desfilis E. Empleo de estrategias de afrontamiento en cuidadores familiares de ancianos con demencia. Rev Esp Geriat Gerontol. 1997; 32(5): 257-69.

13. Larrañaga I, Martín U, Bacigalupe A, Begiristáin JM, Valderrama MJ, Arregi B. Impacto del cuidado informal en la salud y la calidad de vida de las personas cuidadoras: análisis de las desigualdades de género. Gac Sanit 2008;22(5):443-50.

14. Crespo-López M, López-Martínez J. Cuidadoras y cuidadores: el efecto del género en el cuidado no profesional de los mayores. Boletín sobre el Envejecimiento. Perfiles y Tendencias. No. 35. $1^{\text {a }}$ ed. [Internet]. Madrid: IMSERSO; 2008 [consultado 15 de abril de 2016]. Disponible en: http://www.imserso.es/interpresent3/groups/ imserso/documents/binario/boletinopm35.pdf

15. Cerquera-Córdoba AM, Granados-Latorre FJ, Galvis-Aparicio MJ. Relación entre estrato económico y sobrecarga en cuidadores de pacientes con Alzheimer. Rev Virtual Univ Catol del Norte [Internet]. 2014; 42:12737. [consultado 15 de abril de 2016] Disponible en:: http://revistavirtual.ucn.edu.co/index.php/RevistaUCN/ article/view/499/1035

16. Barreto RV, Coral-Ibarra R del C, Campos MS, Gallardo-Solarte K, Ortiz VT. Cuidadores y cuidadoras familiares de personas con enfermedad crónica en Colombia: más similitudes que diferencias. Salud Uninorte Barranquilla (Col.) 2015;31(2):255-265.

17. Figueroa Y. Situación del cuidador y características del cuidado de personas con discapacidad en Santiago de Cali. Rev Chil Salud Pública 2011; 15(2):83-39.

18. Romero-Masa E, Maccausland-Segura Y, Solorzano-Torrecila L. El cuidador familiar del paciente renal y su calidad de vida, Cartagena (Colombia). Salud Uninorte Barranquilla (Col.) 2014;30(2):146-157

19. Ávila-Toscano JH, Vergara-Mercado M. Calidad de vida en cuidadores informales de personas con enfermedades crónicas. Aquichan 2014; 14(3):417-429.

20. Flórez-Torres IE, Montalvo-Prieto A, Herrera-Lian A. Calidad de vida de cuidadores de adultos con accidente cerebrovascular. av enferm. 2010; 28(Especial):52-60.

21. Montalvo-Prieto A, Romero-Massa E, Florez-Torres IE. Percepción de la calidad de vida de cuidadores de niños con cardiopatía congénita Cartagena, Colombia. Investig Educ Enferm 2011; 29(1):9-18. 
22. Garzón-Aponte LA, Pinzón-Rocha ML, Galvis-López CR. Nivel de funcionalidad de los enfermos crónicos y su relación con la calidad de vida de los cuidadores informales, Villavicencio, Colombia, 2011. Enferm Glob. 2014; 13(1):191-200.

23. Romero-Masa E, Montalvo-Prieto A, Flórez-Torres IE. Características de los cuidadores de niños con cardiopatías congénitas complejas y su calidad de vida. av enferm 2010;28(1):39-50

24. Flórez-Torres IE, Montalvo-Prieto A, Herrera-Lían A, Romero-Massa E. Afectación de los bienestares en cuidadores de niños y adultos con enfermedad crónica. Rev Salud Pública (Bogotá). 2010; 12(5):754-64.

25. Montalvo Prieto A, Torres IEF. Características de los cuidadores de personas en situación de cronicidad. Cartagena (Colombia). Salud Uninorte Barranquilla (Col.) 2008;24(2):181-190.

26. Manso ME, Sánchez M, Cuéllar I. Salud y sobrecarga percibida en personas cuidadoras familiares de una zona rural. Clínica y Salud [Internet]. 2013 [consultado 23 de marzo de 2016]; 24(1): 37-45. Disponible en: http:// scielo.isciii.es/scielo.php?script=sci_arttext\&pid=S1130-52742013000100005\&lng=es.

27. Aldana G, Guarino L. Sobrecarga, afrontamiento y salud en cuidadoras de pacientes con demencia tipo Alzheimer. SUMMA Psicológica UST. 2012, 9(1): 5-14

28. Ortiz-Claro YG, Lindarte-Clavijo AA, Jiménez-Sepúlveda MA, Vega-Angarita OM. Características sociodemográficas asociadas a la sobrecarga de los cuidadores de pacientes diabéticos en Cúcuta. Revista Cuidarte 2013; 4(1):459-466.

29. Moreno ME, Nader A, López-Mesa C. Relación entre las características de los cuidadores familiares de pacientes con discapacidad y la percepción de su estado de salud. av. enferm. 2004; 22(1):27-38.

30. Arias RM, Barrera OL, Carrillo GM, Chaparro DL, Sánchez HB, Vargas RE. Cuidadores familiares de personas con enfermedad crónica en las regiones de frontera colombiana: diagnóstico y carga percibida. Rev. Fac. Med. [Internet]. 2014 [consultado 7 de enero de 2012]; 62: 387-397. Disponible en: http://www.revistas.unal.edu.co/ index.php/revfacmed/article/view/39091/50104

31. Ballesteros JE, Rodríguez AM, Cantor M, Peñalosa G, Valcárcel E. Caracterización y percepción de carga de cuidadores familiares de personas con enfermedad crónica en Arauca. Revista Orinoquia. 2015; 19(1): 100-105.

32. Carrillo GM, Chaparro L, Sánchez B. Carga del cuidado en cuidadores familiares de personas con enfermedad crónica en la región Pacífica colombiana. Ciencia y Enfermería 2014 20(2): 83-91.

33. Dueñas E, Martínez MA, Morales B, Muñoz C, Viáfara AS, Herrera JA. Síndrome del cuidador de adultos mayores discapacitados y sus implicaciones psicosociales. Colombia Médica 2006; 37(1):31-38.

34. Chaparro DL, Sánchez HB, Carrillo GG, Barrera OL. ICT as a social support mechanism for family caregivers of people with chronic illness: a case study. Aquichan 2013; 13: 27-40.

35. Republica de Colombia. Departamento Nacional de Estadisticas (DANE). Demografía y Población: Proyecciones de Población. [Internet]. 2016 [citado 28 de abril de 2016]. Disponible en: http://www.dane.gov.co/index.php/ poblacion-y-demografia/proyecciones-de-poblacion

36. Chaparro L, Sánchez B, Carrillo M. Encuesta de caracterización del cuidado de la diada cuidador familiar persona con enfermedad crónica. Rev. cienc. cuidad. 2014; 11(2): 31-45.

37. Zarit SH, Reever KE, Bach PJ. Relatives of the impaired elderly: correlates of feelings of burden. Gerontologist 1980; 20: 649-55.

38. Hayo BK, Velásquez VH, Mayanz SS, Guerra C, Millán KT. Validación en Chile de la Escala de Sobrecarga del Cuidador de Zarit en sus Versiones Original y Abreviada. Rev. méd. Chile 2009; 137: 657-665.

39. Ministerio de Salud de la República de Colombia. Resolución No. 008430, por la cual se establecen las normas científicas, técnicas y administrativas para la investigación en salud. Bogotá D.C.: Ministerio de Salud; 1993.

40. República de Colombia. Ley 911 de 2004, por la cual se dictan disposiciones en materia de responsabilidad deontológica para el ejercicio de la profesión de Enfermería en Colombia. Bogotá D.C.: Congreso de la República de Colombia; 2004.

41. Republica de Colombia. Departamento Nacional de Estadística (DANE). Gran encuesta integrada de hogares 2009. Informe de un grupo científico del DANE. [Internet]. Bogotá: DANE; 2009 [consultado 29 de julio de 2014]. Disponible en: http://www.colombiaaprende.edu.co/html/productos/1685/articles-260524_Destacado. pdf

42. Mesa de Género de la Cooperación Internacional en Colombia. [Internet]. Bogotá: ONU Mujeres; 2013 [consultado 29 de julio de 2014]. Disponible en: http://www.mesadegenerocolombia.org/

43. Carrillo GM, Chaparro L, Sánchez B. Carga del cuidado en cuidadores familiares de personas con enfermedad crónica en la región amazónica de Colombia. Medicina U.P.B. 2014; 33(1): 26-37.

44. Dettinger E, Clarkberg M. Informal caregiving and retirement timing among men and women: Gender and caregiving relationships in late midlife. J Fam Issues 2002; 23: 857-879. 
45. Litwin H. Filial responsibility and informal support among family caregivers of the elderly in Jerusalem: a path analysis. Int J Aging Hum Dev. [Internet]. 1994 [consultado 7 de enero de 2012]; 38:137-51. Disponible en: doi: 10.2190/TLDK-8U5Q-67M8-BMU8

46. Sundström G, Johansson L. Policies and practices in support of family caregivers: Filial obligations redefined in Sweden?. J Aging Soc Policy. [Internet]. 2006 [consultado 7 de enero de 2012]; 18: 7-26. Disponible en: DOI: 10.1300/J031v18n03_02

47. Litwin H, Abramowitz L. Ethnicity and informal support among filial caregivers: Analysis of an Israeli sample. J Cross-Gult Gerontol. [Internet]. 1993 [consultado 7 de enero de 2012]; 8:1-15. Disponible en: doi: 10.1007/ BF00973796

48. Brody EM, Litvin SJ, Hoffman C, Kleban MH. Marital status of caregiving daughters and co-residence with dependent parents. Gerontologist [Internet]. 1995 [consultado 7 de enero de 2012]; 02(35):75-85. Disponible en: doi: 10.1093/geront/35.1.75

49. Yamashita C, Hitomi, Amendola F, Gaspar J, Correia, Martins Alvarenga MR, de CO. Association between social support and the profiles of family caregivers of patients with disability and dependence. Rev. esc. enferm. USP [Internet]. 2013 [consultado 7 de enero de 2012];47:1357-1364. Disponible en: http://dx.doi.org/10.1590/ S0080-623420130000600016

50. Williams KC, Hicks EM, Chang N, Connor SE, Maliski SL. Purposeful normalization when caring for husbands recovering from prostate cancer. Qual Health Res. [Internet. 2014 [consultado 7 de enero de 2012]; 24:306-316. Disponible en: doi: 10.1177/1049732314523842

51. Metzler SE. The Body Gives Way, Things Happen: Older women describe breast cancer with a non-supportive intimate partner. EJON [Internet] 2012 [consultado 7 de enero de 2012];16:64-70. Disponible en: http://dx.doi. org/10.1016/j.ejon.2011.03.006

52. Ocampo JM, Herrera JA, Torres P, Rodríguez JA, Loboa L, García CA. Sobrecarga asociada con el cuidado de ancianos dependientes. ColombMed. 2007; 38:40-46.

53. Rodakowski J, Skidmore E, R., Rogers J, C., Schulz R. Role of Social Support in Predicting Caregiver Burden. Arch Phys Med Rehabil. [Internet]. 2012 [consultado 7 de enero de 2012]; $93: 2229-2236$. Disponible en: https:// www.researchgate.net/publication/229552055_Role_of_Social_Support_in_Predicting_Caregiver_Burden

54. Ohaeri JU. Caregiver burden and psychotic patients' perception of social support in a Nigerian setting. Soc Psych Psych Epid 200; 36: 86-93.

55. Treloar A, Cooper B, Sweeney J, Kelly N. Night respite care: relieving the burden on people caring for relatives with dementia. Nurs Older People [Internet]. 2001 [consultado 7 de enero de 2012]; 13:14-15. DOI: 10.7748/ nop2001.05.13.3.14.c2175

56. Champlain K. Hidden Caregivers: A Literature Review of Young Caregivers in the United States [Tesis doctoral]. University of Pittsburgh; 2012.

57. Bhattacharjee M, Vairale J, Gawali K, Dalal PM. Factors affecting burden on caregivers of stroke survivors: Population-based study in Mumbai (India). Ann Indian Acad Neur [Internet]. 2012 [consultado 7 de enero de 2012];15:113-119. Disponible en: http://www.annalsofian.org/article.asp?issn=0972-2327;year=2012;volume=1 5 ; issue $=2$; ppage $=113$; epage $=119$; aulast $=$ Bhattacharjee; type $=3$

58. Flores E, Rivas E, Seguel F. Nivel de sobrecarga en el desempeño del rol del cuidador familiar de adulto mayor con dependencia severa. Cienc. enferm. [Internet]. 2012 [consultado 7 de enero de 2012]; 18:29-41. Disponible en: http://dx.doi.org/10.4067/S0717-95532012000100004

59. Turner K. Social Support. GeriatrNurs. [Internet]. 1988 [consultado 7 de enero de 2012]; 9: 336-338. Disponible en: http://dx.doi.org/10.1016/S0197-4572(88)80063-6

60. Wilson-Genderson M, Pruchno RA, Cartwright FP. Effects of caregiver burden and satisfaction on affect of older end-stage renal disease patients and their spouses. Psychol Aging. [Internet]. 2009 [consultado 7 de enero de 2012]; 12(24): 955-967. Disponible en: http://www.ncbi.nlm.nih.gov/pmc/articles/PMC2805121/ 